Revista do Departamento de Geografia
Universidade de São Paulo
www.revistas.usp.br/rdg
ISSN 2236-2878

\title{
DINÂMICA GEOMORFOLÓGICA QUATERNÁRIA DA CIMEIRA ESTRUTURAL PERNAMBUCO-ALAGOAS, PLANALTO DA BORBOREMA
}

\author{
QUATERNARY GEOMORPHOLOGICAL DYNAMIC OF \\ PERNAMBUCO-ALAGOAS STRUCTURAL SUMMIT, BORBOREMA HIGHLAND
}

\author{
Évio Marcos Lima \\ Universidade Federal de Pernambuco \\ eviogequa@hotmail.com
}

Antonio Carlos de Barros Corrêa

Universidade Federal de Pernambuco

Drielly Naamma Fonsêca

Universidade Federal de Pernambuco

\begin{abstract}
Resumo: De muitas formas, o período quaternário é substancialmente singular, as oscilações climáticas registradas neste intervalo deixaram mais registros na paisagem geomorfológica que qualquer outro período geológico. O presente trabalho, teve por objetivo principal compreender como evoluiu a dinâmica geomorfológica quaternária em uma região elevada do Planalto da Borborema, Nordeste do Brasil, na área de trabalho escolhida, situa-se o município de Garanhuns. Para tanto, se lançou mão de técnicas de granulometria e morfoscopia, para identificação dos processos de transporte dos grãos e acomodação dos pacotes sedimentares, aliadas à datação por Luminescência Opticamente Estimulada - LOE - para obtenção das idades desses depósitos e realização de coerente alinhamento temporal. Os resultados obtidos, refletiram transições ambientais para períodos secos na faixa dos 30 mil anos, enquanto o momento de entrada no Holoceno acena para um período de maior umidade nos processos morfodinâmicos. Foi ainda possível perceber que o cruzamento entre a circulação climática local e a constituição topográfica da área facilitam a formação de microambientes de exceção ao contexto regional, além de fomentar o avanço de processos químicos, intercalando processos erosivos. Podendo-se concluir que os métodos aplicados foram eficazes no seu propósito.
\end{abstract}

Palavras-Chave: Geomorfologia do Semiárido; L.O.E.; Nordeste do Brasil

\begin{abstract}
In many ways, the Quaternary period is substantially unique, climatic oscillations in this range have left more records in the geomorphological landscape than any other geological period, This work was mainly aimed at understanding how has the dynamic geomorphological Quaternary in an elevated region of the Borborema Highland, Northeast Brazil, the chosen work area, is located in the city of Garanhuns. Therefore, it made use of granulation techniques and morphoscopy for identification of grain transport and accommodation processes of sedimentary packages linked to dating Optically Stimulated Luminescence - OSL - to obtain the ages of these deposits and making coherent time alignment. The results, reflected environmental transitions to dry periods in the range of 30,000 years, while the entry time in the Holocene beckons for a longer period of moisture in morphodynamic processes. It was still possible to see that the cross between the local climate circulation and topographical constitution of the area facilitate microenvironments training exception to the regional context, as well as promoting the advancement of chemical processes, interspersing erosion. It can be concluded that the methods used were effective in their purpose.
\end{abstract}

Keywords: Semi-arid Geomorphology; OSL; Northeast of Brazil 


\section{INTRODUÇÃO}

Sempre foi um dos objetos da geomorfologia compreender a maneira como evolui o modelado superficial da Terra. A evolução das formas do relevo, embasando as paisagens, passa também pela evolução e o desenvolvimento do próprio homem (COLTRINARI, 2011), e por esse prisma, atenção especial é dispensada às modificações geomorfológicas dos últimos 2,6 milhões de anos, período compreendido pelo Quaternário.

De muitas formas, o período quaternário é substancialmente singular, as oscilações climáticas registradas neste intervalo deixaram mais registros na paisagem geomorfológica que qualquer outro período geológico. Tal assertiva pode ser evidenciada na distribuição espacial de depósitos sedimentares resultantes das ações conjuntas de forças como a gravidade, eventos climáticos extremos, oscilações e/ou rupturas climáticas menos abruptas e da ação do próprio homem (SUGUIO, 2010). A dinâmica geomorfológica, bastante ativa do período quaternário, pode ser investigada e reconhecida através de análises de diversos tipos de materiais vestigiais, nos trópicos, no entanto, sobressaem-se os sedimentos coluviais (SUGUIO, op. cit.).

Os processos que elaboram o modelado geram produtos, como resposta do sistema físico, ao input de energia que altera sua dinâmica. Em geral, os resultados dessa interação são os sedimentos superficiais inconsolidados distribuídos pela paisagem, representados por fácies que revelam a dissipação de energia circundante. Desta forma, esses sedimentos representam ferramentas importantes de análise e recomposição dos processos que atuaram no desenvolvimento e controle das taxas de erosão, transporte e sedimentação (CHRISTOFOLETTI, 1980; SAWAKUCHI, et al. 2008). Ainda sob essa perspectiva, Santos (2005, p. 85) afirma que

Cada sub ambiente do sistema deposicional pode ser identificado por sua morfologia, geometria dos depósitos e por uma associação faciológica característica dos processos geomórficos e sedimentares atuantes durante sua formação.

Os depósitos de colúvio têm sua gênese intimamente relacionada à ocorrência de movimentos de massa, estes, por sua vez, ocorrem sob influência de processos gravitacionais gerados, dentre outros, pela ação da água (GOUDIE, 2006). Para Suguio (1973) a forma do grão, os tipos de fraturas e a estrutura dos sedimentos são utilizados, sob a forma de proxies, que permitem remontar os eventos climáticos e o quadro ambiental que funcionaram como agentes desencadeadores da morfogênese dos modelados agradacionais.

O objetivo principal deste trabalho, foi utilizar os depósitos coluviais para reconstruir a dinâmica geomorfológica dos modelados de encosta de um dos setores mais elevados do Planalto da Borborema, Nordeste do Brasil. Para tanto, se lançou mão de técnicas de granulometria e morfoscopia, para identificação dos processos de transporte dos grãos e acomodação dos pacotes sedimentares, aliadas à datação por Luminescência Opticamente Estimulada - LOE - para obtenção das idades desses depósitos e realização de coerente alinhamento temporal, sendo, por fim, realizada uma intersecção entre os aspectos ambientais identificados em um momento pretérito, aos diagnósticos presentes, para a região estudada, a partir de informações climáticas e pedológicas com apoio de componentes gráficos e dados em SIG's.

Tomando-se os sedimentos coluviais como objetos de análise para a compreensão das transformações sofridas pelo relevo ao longo do tempo, destaca-se a primazia metodológica da aplicação de um método de datação que pontue cronologicamente as características ambientais assinaladas pela investigação sedimentar. No caso dos ambientes de encostas tropicais, com ausência de horizontes orgânicos intervenientes entre as camadas coluviais, o método de datação por Luminescência Opticamente Estimulada - LOE - se destaca como uma opção eficiente por seu baixo custo, rapidez e precisão das inferências cronológicas (GREGORY e GOUDIE, 2011). 
O cálculo da idade no método da luminescência opticamente estimulada é feito pela relação Paleodose/Dose Ambiental; em que a paleodose será resultante do acúmulo de radiação ambiental nos grãos dos minerais cristalinos encontrados no depósito sedimentar, oriunda do decaimento dos isótopos de Urânio, Tório e Potássio. Já a Dose Ambiental consiste na taxa de radiação ionizante acumulada pelos mesmos minerais ao longo de um ano de exposição. Segundo Peixoto et al. 2003, p. 02)

Na natureza, a radiação ionizante que atinge os minerais advém da radiação cósmica e de materiais radioativos que se encontram ao seu redor, essencialmente o Urânio, o Tório e seus isótopos radioativos de longa vida, bem como o Potássio, presentes em grande parte dos materiais sedimentares e solos, ao menos como traços, bombardeando-os com radiação $\alpha, \beta$ e $\gamma$.

O método da LOE tem como alicerce a determinação do total de radiação ionizante recebida desde a inumação final dos depósitos sedimentares, isolando-os definitivamente da exposição à luz solar (RENDELL et al. 1994). Após serem adequadamente amostrados sem exposição à luminosidade, a dose acumulada de radiação pode ser mensurada pela excitação, com luz a um determinado comprimento de onda, de um mineral específico da amostra (usualmente quartzo ou feldspato) e pelo monitoramento da luminescência (LOE) emitida pelo mineral em outro comprimento de onda. Não obstante, a quantidade de luz emitida, para uma determinada dose de radiação acumulada no sedimento, varia de amostra para amostra. Essa diferença de sensibilidade verificada entre as amostras é fruto das diferentes origens dos minerais e história de transporte e sedimentação de cada grão. Assim sendo, não pode haver uma curva de calibração fixa entre a intensidade de luminescência emitida e a dose de radiação recebida. Esta curva de calibração, ou curva dose-resposta, precisa ser construída para cada amostra.

Não obstante, a qualidade dos resultados obtidos pelo método de datação depende muito das características e adequação das amostras e dos procedimentos de análise (GUEDES et al., 2013). Em geral, os erros médios no resultado final são da ordem de 5-10\%, embora valores maiores sejam aceitáveis, sobretudo em áreas que não dispõem de uma cronologia prévia para as unidades de deposição quaternária (DULLER, et al. 2003). Assim sendo, esse trabalho lançou mão do protocolo de datação denominado SAR (Single aliquot regenerative protocol) como definido por Murray e Wintle (2000) e aprimorado por Wintle e Murray (2006). Este protocolo é o mais aceito correntemente para a determinação da dose de radiação acumulada em grãos de quartzo.

\section{MATERIAIS E MÉTODOS}

\section{A área de Estudos}

A área de estudos (Figura 1), situa-se no município de Garanhuns-PE, sobre o macrocompartimento morfoestrutural definido por Corrêa et al. (2010) como Cimeira Pernambuco-Paraíba. Este setor delineia-se na paisagem do Planalto da Borborema, de norte para sul, a partir das imediações do Município de São Caetano, Pernambuco, assumindo uma feição topográfica marcada pelas cimeiras planas recobertas por espesso manto de argissolos e neossolos, cuja elevação varia de 600 a 700 metros. O único ressalto estrutural notável deste macro-compartimento que pode ser designado de "planalto" stricto senso é o patamar erosivo de Garanhuns. Constitui-se numa das superfícies mais elevadas da Borborema (900-1.000 metros) e está estruturado numa faixa de quartzitos, orientada, grosso modo, E-W, relacionada ao Complexo Belém do São Francisco (Mesoproterozóico). 


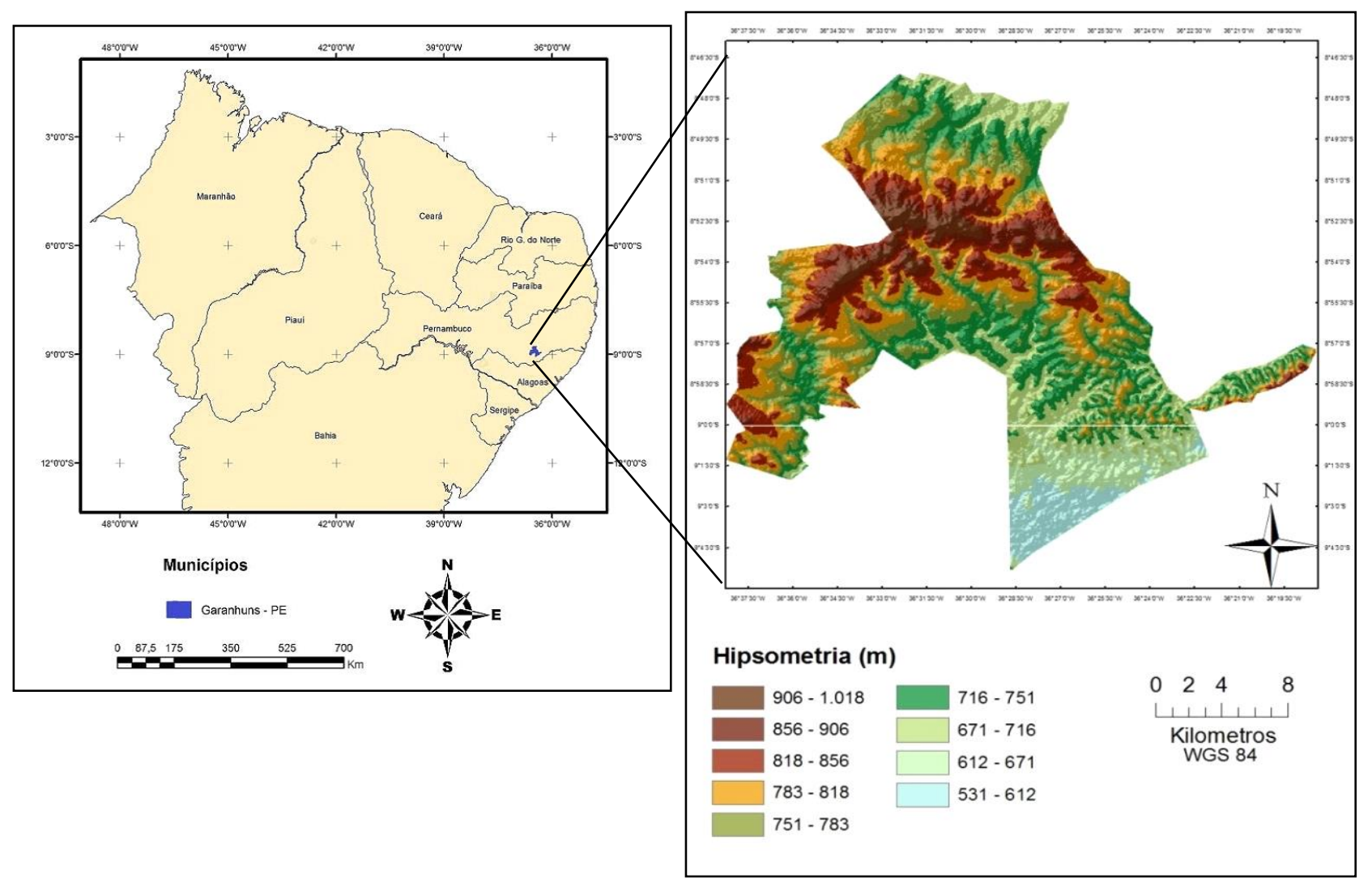

Figura 1: Localização da área de estudos em um contexto regional. Fonte: Autor.

Este setor do Planalto da Borborema é exemplo de como a complexa teia de processos físicos/ambientais, atuando em intensidades variantes ao longo do tempo, podem transformar e influenciar a paisagem e a dinâmica ambiental de determinada porção da superfície da terra. Na área de estudo, a configuração do contexto estrutural, à retaguarda das principais zonas de cisalhamento que atravessam a região, levou à elaboração de uma paisagem geomorfológica marcada por um conjunto de morros e colinas dissecadas, nas encostas dos quais as variações dos regimes hídricos associadas ao espesso manto de regolito resultou na formação de diversas cabeceiras de drenagem de ordem zero (alvéolos não canalizados) e de primeira ordem adaptadas a cicatrizes erosivas préexistentes com até 150 metros de incisão (CORREA et al., 2010).

\section{Análise dos Sedimentos}

As análises sedimentológicas objetivaram a caracterização qualitativa e quantitativa dos sedimentos, bem como se constituíram em uma etapa de interpretação dos aspectos deposicionais para a elaboração das seções verticais e reconstrução morfoestratigráfica. Buscando caracterizar os processos geomorfológicos e a constituição dos sedimentos das unidades geomorficas estudadas, foram realizadas as análises para a definição da granulometria e morfoscopia dos depósitos, permitindo assim confeccionar, a partir da aplicação dos parâmetros de Folk e Ward (1957), gráficos e tabelas que representem a identidade deposicional dos modelados agradacionais amostrados.

Ainda de acordo com Suguio (2002), a análise granulométrica busca estabelecer o tamanho das partículas em sedimentos detríticos, este é um parâmetro textural primordial para a análise das propriedades físicas dos sedimentos siliciclásticos, permitindo a suaclassificação em rudáceos, arenáceos e lutáceos. Por seu turno, a análise morfoscópicados grãos é baseada no método visual de comparação entre a projeção máxima do contorno da partícula e um conjunto de imagens cujo arredondamento foi previamente calculado de acordo com o procedimento descrito por Tucker (1996). Assim, a análise morfoscópica dos sedimentos além de possibilitar a determinação do grau de arredondamento, esfericidade, opacidade e heterogeneidade mineralógica do material, permite ainda definir qualitativamente os processos e ambientes deposicionais a eles associados. 
Para fins de condução de análise granulométrica e morfoscópica dos sedimentos coluviais, foram retiradas sub-amostras de cem gramas, essas amostras passam a imersão em uma solução de vinte gramas de hexametafosfato para cada litro de água, a fim de que sejam eliminados os argilominerais, posteriormente o material é ressecado artificialmente à temperatura de sessenta graus Celsius durante vinte e quatro horas; Após esse processo o material é peneirado em agitador artificial e são selecionados os grãos de acordo com os níveis fracionários das peneiras, desde $2 \mathrm{~mm}$ (cascalho), $1 \mathrm{~mm}, 0.5 \mathrm{~mm}, 0.25 \mathrm{~mm}, 0.125 \mathrm{~mm}, 0,063 \mathrm{~mm}$, e 0,038 . O material analisado sob exposição à lupa óptica foi o que se encaixou na fração.

\section{Identificação das Unidades Geomorfológicas}

Os procedimentos metodológicos adotados neste trabalho tiveram por ponto de partida a identificação das unidades geomorfológicas que integram a área de estudos. Essa identificação teve como fundamento os compartimentos definidos por Lima (2014), com base em seu mapeamento geomorfológico para a região de Garanhuns. A compartimentação do relevo para a área de estudo proposta por Lima (2014), definiu o seguinte conjunto de formas: Áreas de Topo; subdivididas em dois patamares. Estas superfícies mais elevadas estão marcadas por topos amplos e suavemente convexos, apresentando incisões lineares, a partir dos pontos de ruptura entre a cimeira e a encosta, algumas ajustadas a cicatrizes erosivas de paleovoçorocas. Áreas pedimentares, semelhante à unidade anterior, esta, também se apresenta organizada em dois patamares, sendo o primeiro mais atacado pelos processos superficiais apresentando evidências de dissecação por erosão linear intensificada pelas formas de ocupação urbana ora em expansão. Assim sendo, a escolha dos pontos de coleta (Figura 2) se baseou na distribuição dos pacotes mais significativos de sedimentação coluvial. Estes foram observados junto às faixas de transição dentre os patamares supracitados, mormente associados aos sopés de encostas e níveis de base locais, onde as condicionantes ambientais facilitaram o desenvolvimento de espaços de acomodação ao longo das concavidades basais das encostas. As coletas de material foram orientadas segundo as descontinuidades encontradas nos perfis abertos nos depósitos coluviais, onde se assume que tais estruturas de solução de continuidade, discerníveis nas seções estratigráficas, caracterizam diferentes eventos e processos de deposição. Desta forma, os dois pontos de coleta podem ser caracterizados da conforme a seguinte descrição:

- Ponto 1 (VRM): O ponto VRM está situado a uma altitude de $767 \mathrm{~m}$ de elevação, na faixa de transição topográfica entre a cimeira e o pedimento dissecado à $\mathrm{S} / \mathrm{SE}$, caracterizando o início da encosta referente a descida da superfície elevada da Borborema (Figura 3). Neste ponto, foram recolhidas 4 amostras em um perfil com exposição de 2 metros de altura, com medição feita do ponto superficial ao encontro com a rocha mãe ou, como neste caso, com a frente de intemperismo. As amostras foram colhidas a 0,5m, $1 \mathrm{~m}, 1,30 \mathrm{~m}$ e 1,90 m da superfície.

Nesta seção estratigráfica observam-se planos de deposição bem discerníveis entre as unidades deposicionais. Há pelo menos 4 camadas sedimentares depositadas ao longo do perfil. As duas superiores apresentam areias grossas e a distribuição de clastos dispersos, flutuando aleatoriamente na matriz, com uma diferenciação muito sensível entre o primeiro e o segundo pacotes. Abaixo destes, ocorre uma linha de seixos a $130 \mathrm{~cm}$ de profundidade. Em seguida, a unidade sedimentar basal, apresenta uma granulometria mais fina, maior compactação e a distribuição menos constante de fenoclastos ainda dispersos de forma aleatória na matriz.

- Ponto 2 (BV): O ponto BV (Figura 4) encontra-se a uma altitude de 846m. Local que marca a zona de transição entre dois patamares mais elevados que integram o relevo da área de estudo - Cimeira e Cimeira Somital (LIMA, 2016). A seção vertical onde foi realizada a coleta de sedimentos localiza-se ao sopé das elevações com face voltada a $\mathrm{N}$ do divisor principal.

Apesar da localização do pacote coluvial em uma concavidade basal ao sopé de uma elevação, a espessura identificada de sedimentos foi de apenas $1,10 \mathrm{~m}$, desde a superfície até a frente de intemperismo. Pedologicamente, a área configura uma zona de transição entre a ocorrência de um latossolo amarelo e um neossolo regolítico (EMBRAPA, 2006). 


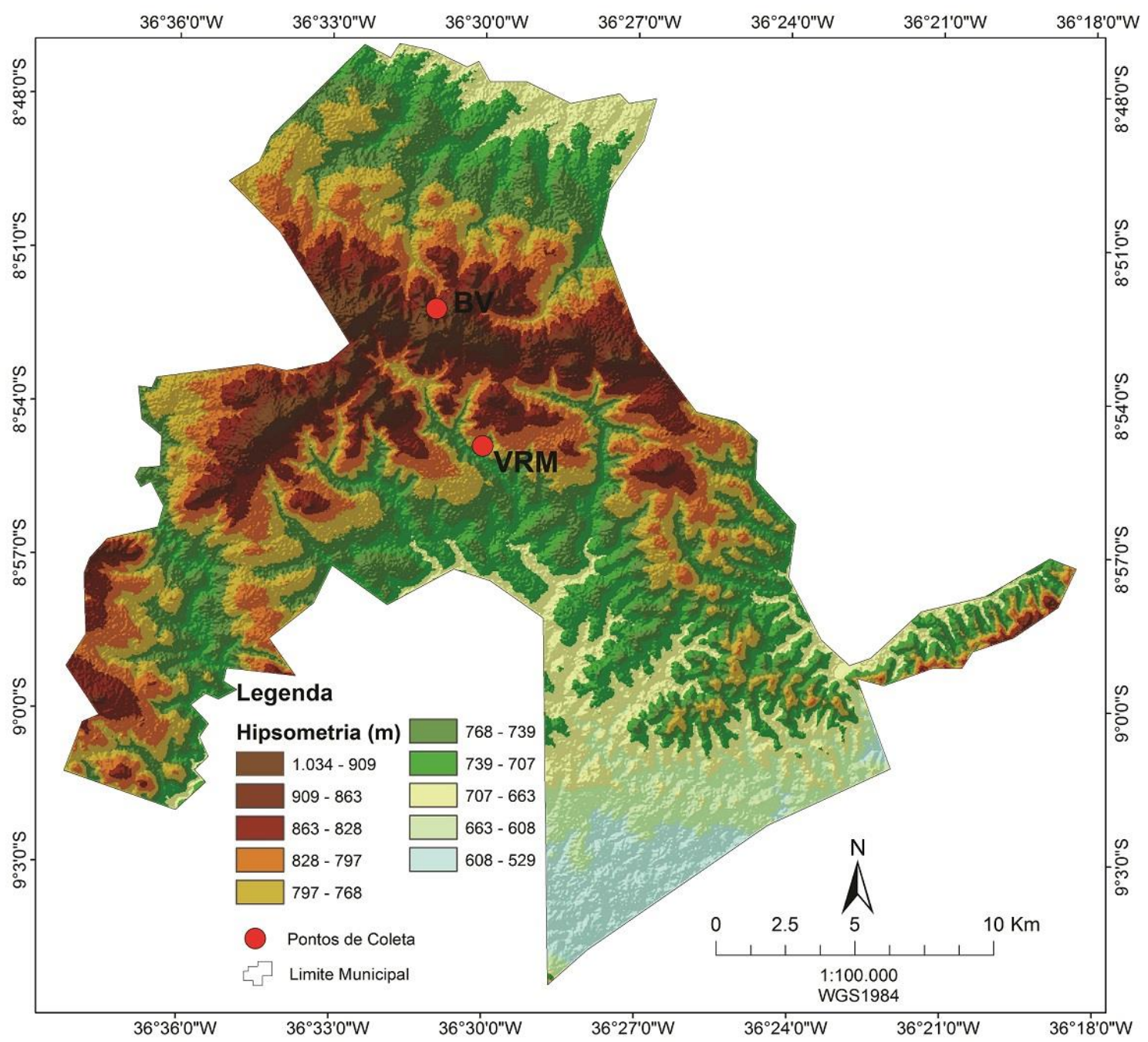

Figura 2: Mapa de localização dos pontos de coleta, elaborado pelos autores a partir da base de dados SRTM (Shuttle Radar Topographic Mission). Fonte: Autor.

O perfil BV apresenta dois planos de deposição bem definidos, entretanto, a variação granulométrica permite a identificação de, pelo menos, 4 intervalos deposicionais bem discerníveis. À altura da unidade BV-45, a fábrica é marcada pela presença de fenoclastos "flutuando" aleatoriamente em uma matriz de areais médias a finas. O nível BV-100 apresenta estrutura mais homogênea, e um maior grau de compactação próximo à frente de intemperismo.

\section{Datação por LOE}

Os métodos de datação absoluta de sedimentos, são formas precisas de identificar as idades de certos fenômenos identificados em diversos materiais detríticos. No método LOE, seguindo o protocolo de Regeneração de Alíquota Única - SAR em inglês - a precisão aumenta devido à diminuição do ruído no processo de fotoionização do quartzo (SCHIMIDT, et al., 2013) atuando em pulsos de luz verde na carga potencial, reclusa no momento da deposição. A técnica consiste na emissão de energia ao sistema em que o elétron está inserido, essa energia, radiativa, vai gerar o decaimento dos elétrons recombinados, liberados em forma de fóton (CLARKE et al., 1999; MURRAY e WINTLE, 2000). 


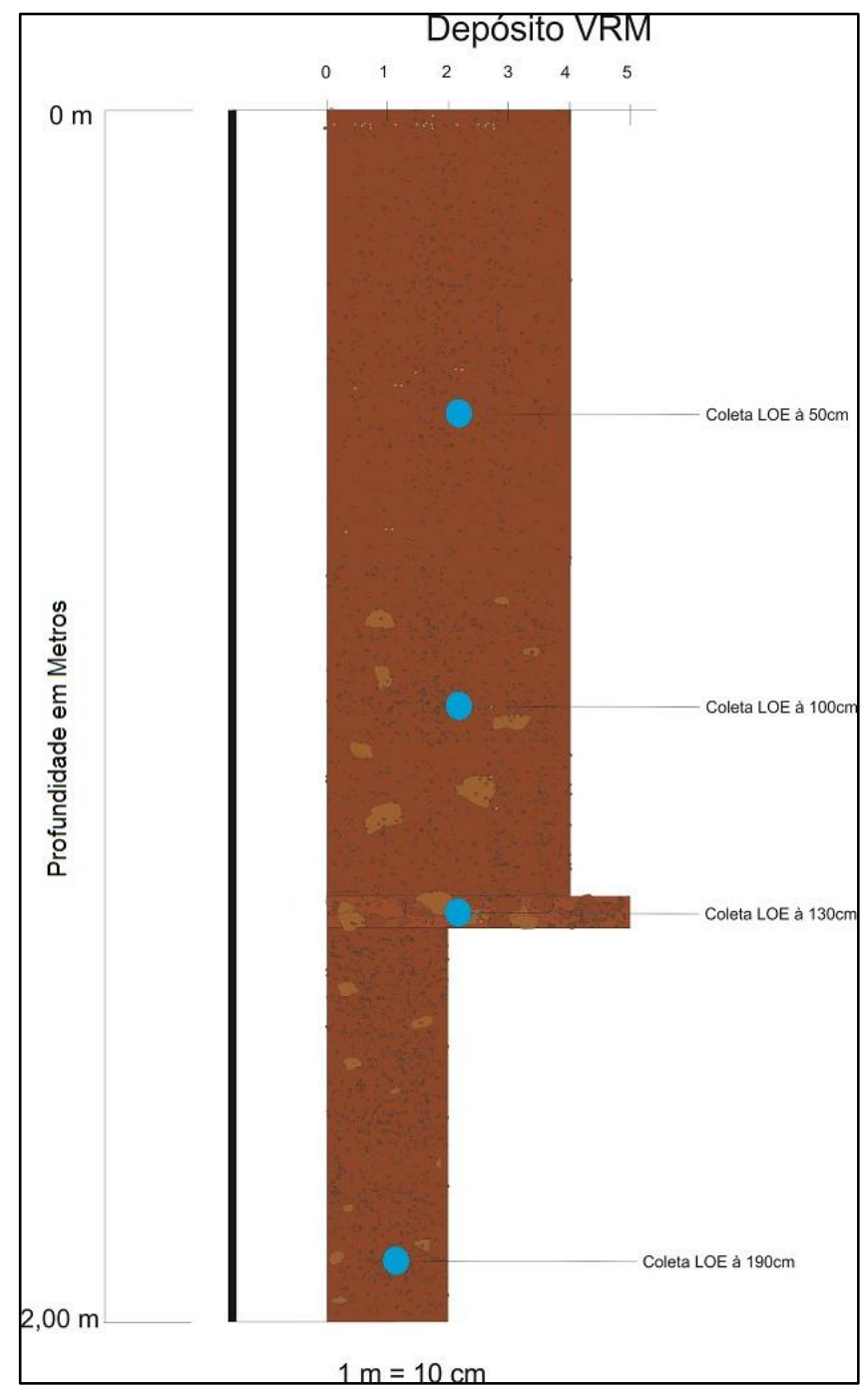

Figura 3: Perfil de sequência sedimentar do depósito VRM. Fonte: Autor.

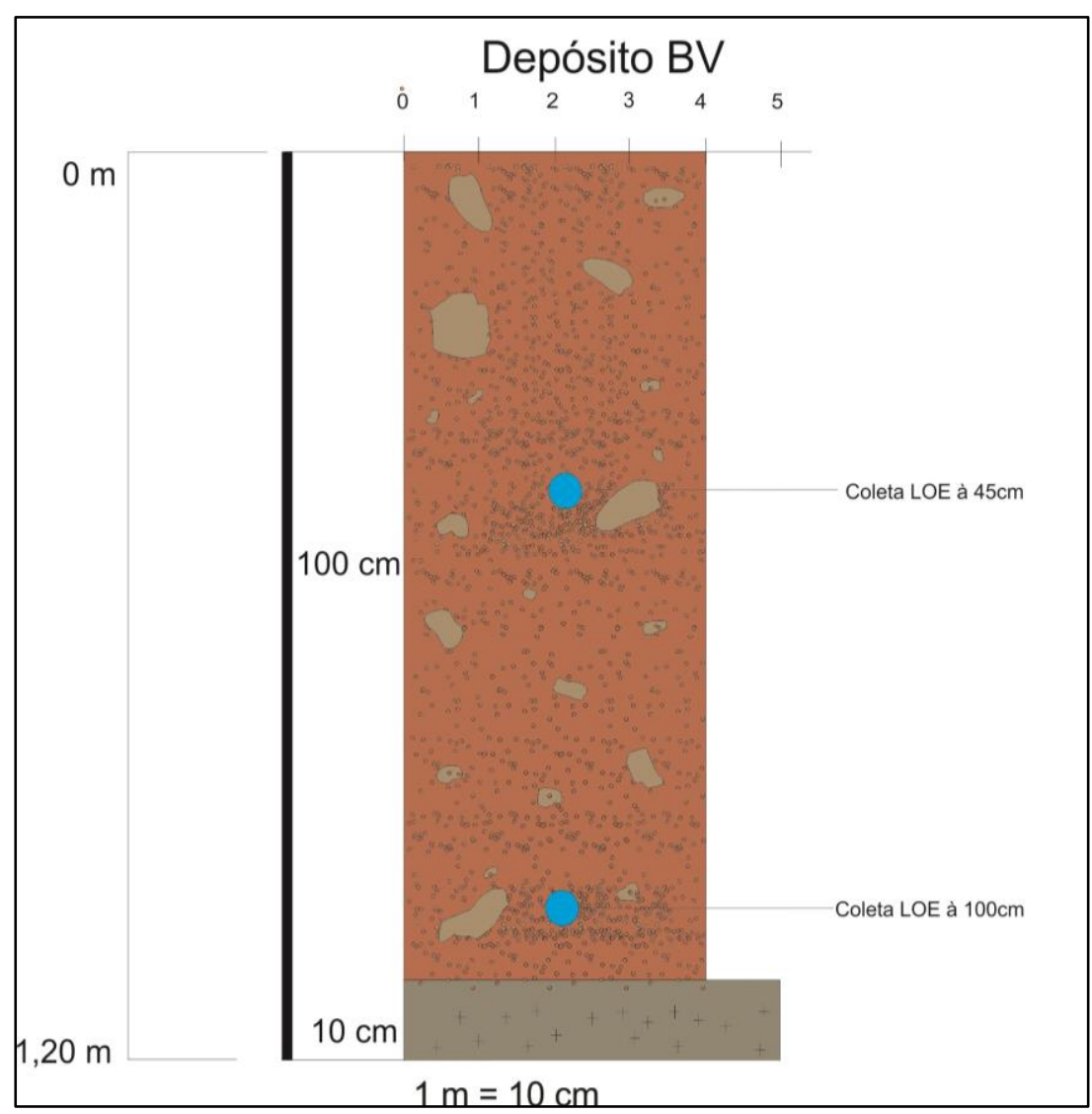

Figura 4: Ilustração do perfil BV. Fonte: Autor. 
Segundo método proposto por Aitken (1998) a preparação das amostras de quartzo para o protocolo SAR envolve a imersão em $\mathrm{H} 2 \mathrm{O} 2$ para a retirada que quaisquer resquícios de matéria orgânica; uma solução de $10 \%$ de $\mathrm{HCl}$ para a dissolução dos carbonatos. Em seguida é adicionada uma mistura a $40 \%$ de HF para remoção do feldspato restante. No protocolo SAR, a curva de calibração, bem como a medida da luminescência natural, é realizada um uma única sub-amostra ou alíquota. Desta forma, evita-se o uso de mais de uma alíquota que de fato apresentariam diferentes sensibilidades da LOE. Este procedimento tem demonstrado que o resultado final é mais confiável e menos variável. Outra característica do protocolo é a realização da medição da luminescência após submeter as alíquotas a pequenas doses de radiação a cada ciclo de leitura da LOE. Essa rotina é usada como maneira de corrigir a variação de sensibilidade da LOE da alíquota. Assim, o resultado final de uma amostra de sedimento é composto pela média do resultado de diversas alíquotas (em geral 15 alíquotas), sendo em geral bastante confiável.

Para fins de datação pelo método LOE-SAR as amostras coletadas de seis níveis estratigráficos representativos em ambas as áreas de estudo foram enviadas ao laboratório "Datação: Comércio e Serviços, LTDA" na cidade de São Paulo - SP. As amostras foram identificadas no laboratório como 4071 até 4076, para VRM-190, VRM-130, VRM-100, VRM-50, BV-100 e BV-45, respectivamente.

\section{RESULTADOS E DISCUSSÕES}

Assim, os resultados das análises granulométricas nos permitem tecer inferências sobre os tipos de processos de transporte e deposição, refletindo as características ambientais da região nos momentos de ocorrência destes fenômenos. Posteriormente, atribui-se a cada nível sedimentar cujo processo formativo foi pré-estabelecido uma idade LOE-SAR, que equivale o período em que o evento deposicional ocorreu ao longo do Quaternário tardio. Em seguida os episódios deposicionais são confrontados com outros cenários obtidos por meio de reconstrução das condições paleoambientais do Planalto da Borborema, o que por fim nos permite inferir se o comportamento deposicinal na unidade morfoestrutural da Cimeira Pernambuco-Alagoas foi semelhante ou diverso daqueles já estabelecidos para outros setores do Planalto.

\section{Análises Granulométricas}

A análise da granulometria resultou na construção de tabelas e gráficos que aportaram uma síntese ao procedimento analítico aplicado, permitindo inferências sobre os mecanismos de transporte nas encostas. A tabela de distribuição granulométrica por peso (Tabela 1) expõe a distribuição dos materiais, por diâmetro do grão, contidos nos depósitos coluviais amostrados, enquanto a tabela morfo sedimentar (Tabela 2) apresenta os dados relacionados às formas identificadas dos grãos analisados. O grau de seleção e outros parâmetros físicos foram correlacionados a processos ambientais associados ao transporte e deposição (Figuras 5 e 6).

Tabela 1: Tabela apresentando a distribuição granulométrica por peso, para amostragem de $100 \mathrm{~g}$ de material.

\begin{tabular}{|c|c|c|c|c|c|c|c|c|}
\hline \multicolumn{9}{|c|}{ Tabela Granulométrica } \\
\hline Amostra & $\begin{array}{l}>1.00 \\
\mathrm{~mm}\end{array}$ & $0.5 \mathrm{~mm}$ & $0.250 \mathrm{~mm}$ & $0.125 \mathrm{~mm}$ & $0.063 \mathrm{~mm}$ & $0.038 \mathrm{~mm}$ & $\begin{array}{l}<0.038 \\
\mathrm{~mm}\end{array}$ & $\begin{array}{l}\text { Peso } \\
100 \%\end{array}$ \\
\hline VRM - 50 & $31.448 \mathrm{~g}$ & $22.226 \mathrm{~g}$ & $21.466 \mathrm{~g}$ & $13.759 \mathrm{~g}$ & $6.508 \mathrm{~g}$ & $3.833 \mathrm{~g}$ & $0.917 \mathrm{~g}$ & $100.006 \mathrm{~g}$ \\
\hline $\begin{array}{l}\text { VRM - } \\
100\end{array}$ & $33.041 \mathrm{~g}$ & $22.904 \mathrm{~g}$ & $20.586 \mathrm{~g}$ & $12.487 \mathrm{~g}$ & $6.650 \mathrm{~g}$ & $4.438 \mathrm{~g}$ & $0.138 \mathrm{~g}$ & $100.004 \mathrm{~g}$ \\
\hline $\begin{array}{l}\text { VRM - } \\
130\end{array}$ & $28.977 \mathrm{~g}$ & $20.749 \mathrm{~g}$ & $24.255 \mathrm{~g}$ & $13.979 \mathrm{~g}$ & $8.781 \mathrm{~g}$ & $3.255 \mathrm{~g}$ & $0.284 \mathrm{~g}$ & $100.008 \mathrm{~g}$ \\
\hline $\begin{array}{l}\text { VRM - } \\
190 \\
\end{array}$ & $36.252 \mathrm{~g}$ & $19.955 \mathrm{~g}$ & $19.170 \mathrm{~g}$ & $11.995 \mathrm{~g}$ & $8.946 \mathrm{~g}$ & $3.595 \mathrm{~g}$ & $0.155 \mathrm{~g}$ & $100.008 \mathrm{~g}$ \\
\hline BV - 45 & $59.598 \mathrm{~g}$ & $21.952 \mathrm{~g}$ & $11.098 \mathrm{~g}$ & $3.411 \mathrm{~g}$ & $3.592 \mathrm{~g}$ & $0.500 \mathrm{~g}$ & $0.020 \mathrm{~g}$ & $100.004 \mathrm{~g}$ \\
\hline BV -100 & $61.9 \mathrm{~g}$ & $19.7 \mathrm{~g}$ & $9.2 \mathrm{~g}$ & $4.4 \mathrm{~g}$ & $2.5 \mathrm{~g}$ & $1.8 \mathrm{~g}$ & $0.061 \mathrm{~g}$ & $100.010 \mathrm{~g}$ \\
\hline
\end{tabular}

Fonte: Autor. 
Tabela 2: Tabela apresentando a distribuição sedimentar por forma dos grãos, variando em função do ambiente de transporte e deposição.

\begin{tabular}{|c|c|c|c|c|c|c|c|c|c|c|}
\hline \multicolumn{11}{|c|}{ Tabela Morfo Sedimentar } \\
\hline \multirow{2}{*}{ Amostra } & \multicolumn{3}{|c|}{ Esfericidade } & \multicolumn{3}{|c|}{ Arredondamento } & \multicolumn{2}{|c|}{ Textura } & \multicolumn{2}{|c|}{ Opacidade } \\
\hline & Discoidal & Esférico & Prismoidal & Anguloso & Intermediário & Arredondado & Polida & Fosco & Transparente & Opaco \\
\hline VRM - 50 & $33 \%$ & $51 \%$ & $16 \%$ & $48 \%$ & $27 \%$ & $25 \%$ & $87 \%$ & $13 \%$ & $42 \%$ & $58 \%$ \\
\hline VRM - 100 & $38 \%$ & $50 \%$ & $12 \%$ & $39 \%$ & $31 \%$ & $30 \%$ & $71 \%$ & $29 \%$ & $40 \%$ & $60 \%$ \\
\hline VRM - 130 & $52 \%$ & $33 \%$ & $15 \%$ & $30 \%$ & $31 \%$ & $39 \%$ & $75 \%$ & $25 \%$ & $47 \%$ & $53 \%$ \\
\hline VRM - 190 & $29 \%$ & $40 \%$ & $21 \%$ & $40 \%$ & $23 \%$ & $37 \%$ & $79 \%$ & $21 \%$ & $51 \%$ & $49 \%$ \\
\hline BV - 45 & $41 \%$ & $26 \%$ & $23 \%$ & $22 \%$ & $32 \%$ & $46 \%$ & $80 \%$ & $20 \%$ & $39 \%$ & $61 \%$ \\
\hline BV - 100 & $35 \%$ & $41 \%$ & $24 \%$ & $30 \%$ & $35 \%$ & $35 \%$ & $73 \%$ & $27 \%$ & $47 \%$ & $53 \%$ \\
\hline
\end{tabular}

Fonte: Autor.

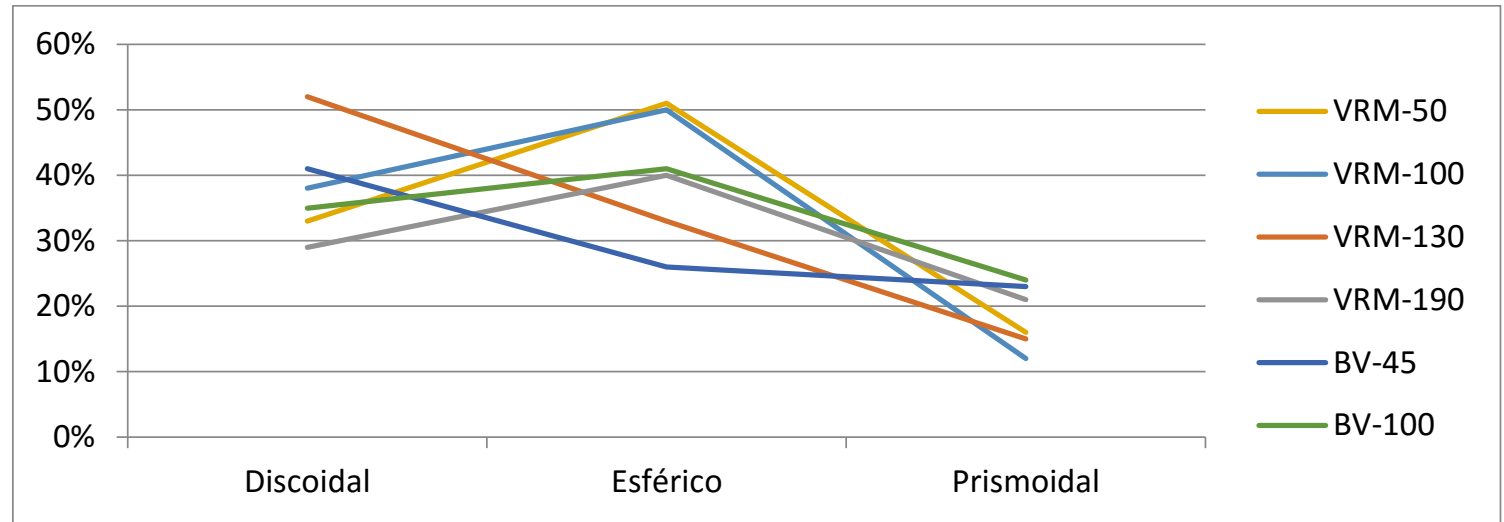

Figura 5: Gráfico de Esfericidade dos grãos amostrados. Fonte: Autor.

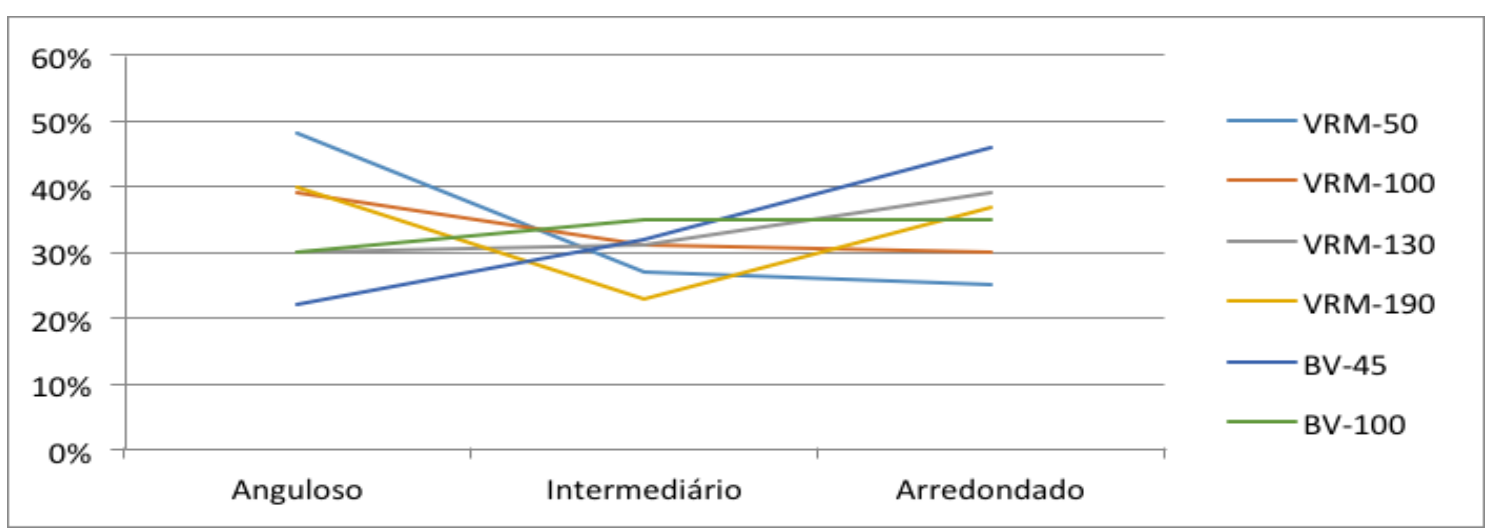

Figura 6: Gráfico de esfericidade das amostras sedimentares analisadas. Fonte: Autor.

A distribuição dos níveis de arredondamento dos grãos nas amostras VRM - 100 e VRM 130 indica a semelhança dos processos ambientais durante o período datado, enquanto a predominância de sedimentos arredondados nas amostras BV - 45 e BV - 100 refletem a presença de água no sistema de transporte (SUGUIO, 1973), dado corroborado pelas informações de opacidade para essas amostras. Estas informações indicam transições ambientais entre um momento mais seco, por volta de 30.000 anos A.P., sob a forma de uma flutuação climática pontual, e um momento mais úmido, entre 8000 e 5000 anos A.P., seguindo o estabelecimento do Holoceno, com a maior presença de umidade na atmosfera.

As amostras da área VRM apontam para a ocorrência de um fluxo com menor capacidade de transporte, com predominância de grãos discoidais. Por sua vez, as amostras da área BV apontam para a ação de fluxos de intensidade oscilante, com ativação e declínio acelerados, o que se evidencia pela angulosidade elevada dos grãos analisados.

A análise granulométrica permitiu identificar cenários de pluviosidade concentrada e elevada, permitindo inferir sobre o desenvolvimento de eventos extremos de precipitação, relacionados à transição pleistoceno/holoceno, para as superfícies mais elevadas, ao Norte. Por outro lado, pelo 
volume do perfil analisado, bem como sua distribuição granulométrica, é possível perceber que as vertentes à $\mathrm{S}$-SE da área de estudos permaneceram sob regime de precipitação similar durante o UMG, tendo ligeiros pontos de perturbação, registrados pela ocorrência de linhas de pedras (stonelines), além da perceptível variação de granulometria entre os dois pacotes que baseiam a sequência (VRM-130 e VRM-190).

A partir do início do Holoceno, a atuação da ZCIT (Zona de Convergência Intertropical) se fez mais presente nas regiões a oeste do Planalto da Borborema, enquanto as vertentes orientais do Planalto passaram a receber a atuação mais intensa das massas oceânicas através da maior penetração dos DOL (Distúrbios Ondulatórios de Leste) (AMORIM, 2015). A percepção das mudanças ocorridas na paisagem, podem ser constatadas pela comparação entre os processos e ambientes de sedimentação atuais, com predomínio da ação do fluxo superficial concentrado e ravinamentos, pretéritos, sob a égide dos fluxos gravitacionais de encosta e acumulação coluvial sobre os knick-points.

\section{Datação por Luminescência Opticamente Estimulada - LOE.}

A partir da datação por LOE foi possível estabelecer 4 momentos distintos (Tabela 3) de elaboração dos modelados coluviais na área do Patamar Erosivo de Garanhuns. Estas idades apontam para a constatação de que as modificações ambientais registradas nas encostas como sedimentação coluvial remontam aos últimos 40.000 anos. A datação obtida neste trabalho também corroborou algumas constatações geocronológicas realizadas em trabalhos recentes (SILVA, 2007; GALVÃO, 2012; LIRA, 2014) para os sedimentos inconsolidados que estruturam modelados de agradação no semiárido do NE do Brasil. O fato dos sedimentos ora analisados serem cronocorrelatos aos de outros estudos realizados na região reforçam a confiabilidade dos resultados encontrados.

A Tabela 4 mostra as concentrações medidas dos isótopos radioativos 232Th, 238U+235U, $40 \mathrm{~K}$ utilizados para calcular a dose anual que é a taxa equivalente à quantidade de radiação ambiental acumulada pelos sedimentos durante o período de um ano.

A amostra mais antiga da área resultou em uma idade de 42.000 anos AP. Regionalmente este momento é tido como um intervalo de maior umidade anterior ao Último Máximo Glacial - UMG caracterizado por uma flutuação climática de curto prazo identificada como um interestadial. Apesar da proximidade ao front de intemperismo - $190 \mathrm{~cm}$ de profundidade - a granulometria deste nível situou-se majoritariamente entre os intervalos de 500 e 250 um, sem incorporação de fenoclastos da base, o que reforça a ideia de que se trata de uma unidade alóctone.

O segundo evento identificado foi observado a partir de duas amostras coletadas a $130 \mathrm{~cm} \mathrm{e}$ $100 \mathrm{~cm}$ de profundidade, respectivamente, no ponto VRM. Na primeira foi observada uma cascalheira interpretada como linha de pedras - stone-line - derivada de possível flutuação climática local pontuando a modificação ambiental resultante da transformação relacionada à entrada no último máximo glacial.

Tabela 3: Idades aproximadas para as amostras coletadas em Garanhuns. Fonte: Laboratório Datação.

\begin{tabular}{|c|c|c|c|}
\hline Amostra & Dose Anual & Dose Acumulada & Idade Obtida \\
\hline VRM - $190 \mathrm{~cm}$ & $2720+-130$ & 114,1 & \multirow{2}{*}{$42000+-4070$} \\
\hline \multicolumn{2}{|c|}{ DESVIO PADRÃO } & 8,5 & \\
\hline VRM - $130 \mathrm{~cm}$ & $3200+-215$ & 107,5 & \multirow{2}{*}{$33.580+-3.920$} \\
\hline \multicolumn{2}{|c|}{ DESVIO PADRÃO } & 13,1 & \\
\hline VRM - $100 \mathrm{~cm}$ & $3.260+-190$ & 99,6 & \multirow{2}{*}{$30.550+-3.320$} \\
\hline \multicolumn{2}{|c|}{ DESVIO PADRÃO } & 9,2 & \\
\hline VRM - $50 \mathrm{~cm}$ & $2.100+-85$ & 14,8 & \multirow{2}{*}{$7.060+-640$} \\
\hline \multicolumn{2}{|c|}{ DESVIO PADRÃO } & 2,9 & \\
\hline BV - $100 \mathrm{~cm}$ & $1.420+-80$ & 12,2 & \multirow{2}{*}{$8.580+-910$} \\
\hline \multicolumn{2}{|c|}{ DESVIO PADRÃO } & 2,2 & \\
\hline BV $-45 \mathrm{~cm}$ & $1.250+-75$ & 6,9 & \multirow{2}{*}{$5.540+-620$} \\
\hline \multicolumn{2}{|c|}{ DESVIO PADRÃO } & 1,6 & \\
\hline
\end{tabular}


Tabela 4: Concentrações dos isótopos radiativos para Urânio (U) e Tório (Th) em partes por milhão e o percentual de Potásio (K). Fonte: Laboratório Datação.

\begin{tabular}{|c|ccc|}
\cline { 1 - 2 } AMOSTRA & TH (ppm) & U (ppm) & K (\%) \\
\cline { 1 - 2 } $\mathbf{4 0 7 1}$ & $17,605+-0,634$ & $4,284+-0,233$ & $0,134+-0,019$ \\
$\mathbf{4 0 7 2}$ & $21,070+-0,759$ & $4,464+-0,443$ & $0,287+-0,042$ \\
$\mathbf{4 0 7 3}$ & $18,311+-0,659$ & $4,612+-0,256$ & $0,512+-0,074$ \\
$\mathbf{4 0 7 4}$ & $13,210+-0,476$ & $2,854+-0,092$ & $0,178+-0,026$ \\
$\mathbf{4 0 7 5}$ & $6,450+-0,232$ & $1,360+-0,020$ & $0,383+-055$ \\
$\mathbf{4 0 7 6}$ & $6,964+-0,251$ & $1,729+-0,178$ & $0,084+-0,012$ \\
\hline
\end{tabular}

Segundo Corrêa (2001) as cascalheiras intermediárias como aquelas encontradas na seção VRM, não apenas confirmam que sua gênese está atrelada ao retrabalhamento do material encosta abaixo, como também indicam que essas camadas de sedimento rudáceo atestam a ocorrência de eventos deposicionais discretos e rápidos. Tal interpretação afasta a noção de que essas estruturas sejam remanescentes de paleo-pavimentos detríticos.

A existência de latossolos na mesma região do ponto de coleta pode ser um reflexo da mudança ambiental ocorrida com a chegada do holoceno e o aumento da precipitação ao longo dos últimos 10 mil anos, o que coincide com o período interglacial onde, segundo Suguio (2010) as temperaturas mais elevadas influenciariam positivamente as médias de precipitação, sobretudo nas regiões tropicais, acentuando, assim, o intemperismo químico e a pedogênese; aliado à elevação da temperatura, decorre o aumento na variedade biológica.

O momento de transição entre o período Pleistoceno e o Holoceno foi observado na amostra BV-100. Por outro lado, idades na faixa dos 8 mil anos A.P. marcam um momento mais úmido compreendido no Holoceno Superior. O recuo das camadas de gelo nas latitudes médias favoreceu a precipitação nas zonas tropicais, e esses ambientes começaram a se tornar gradativamente mais úmidos. $\mathrm{Na}$ área de estudo há diferenciação no processo de intemperismo, as áreas de encostas, submetidas a precipitações mais intensas, passam a perder material e a sedimentação de fundo de vale começa a se desenvolver.

O depósito BV apresenta idade típica das elaborações recentes de sedimento de encosta do Agreste Pernambucano (SILVA, 2007), com período de elaboração restrito ao holoceno. Percebe-se ainda a ação da influência do relevo local em face dos sistemas climáticos ora operantes. Este fator, localmente definido pelas características topográficas do Patamar erosivo de Garanhuns, dominado pela orientação NE-SW dos vales, certamente funcionou como barreira orográfica, em escala subregional, capturando a umidade das massas de ar provenientes do oceano.

O que difere este espaço com relação à temporalidade da elaboração do seu modelado, em relação aos setores situados mais a oeste de Pernambuco e do semiárido em geral, diz respeito ao fato de que, durante o último máximo glacial (UMG), a atuação morfogenética do clima se mostrou intensificada em relação à fachada atlântica do Planalto da Borborema. Essa dessincronia na ação da morfogênese das encostas pode ser verificada pelos estudos realizados no Sertão de Pernambuco (LIRA, 2014; GALVÃO, 2012) cujas idades encontradas remontam ao final do pleistoceno, com ênfase para o UMG.

Dada às características dos sedimentos e dos depósitos investigados neste estudo, onde fica exposto a configuração de um cenário de umidade construído, sobretudo ao longo do Holoceno, neste ínterim, sugere-se uma maior penetração da umidade de Leste fomentando o desenvolvimento de perfis mais aprofundados e de gênese holocênica - ponto $\mathrm{BV}$-, solos com pedogênese mais complexa e padrões de transporte relacionados a tração hidráulica.

Todos esses fatores estão relacionados fundamentalmente às mudanças no comportamento dos processos ambientais, contudo, é salutar compreender a relevância da constituição topográfica regional pois esta, exerce, em escala local, um controle na distribuição pluviométrica entre as áreas ao Sul e ao Norte do Patamar Erosivo de Garanhuns de orientação geral E-W. O reflexo dessa 
distribuição é notável sobre a repartição espacial das coberturas pedológicas na área (Figura 7), separando solos com pedogênese mais intensa, como argissolos latossolos (EMBRAPA, 2006), juntos às zonas de cimeira e suas vertentes à $\mathrm{S} / \mathrm{SE}$, dos Neossolos, nas superfícies aplainadas ao Norte.

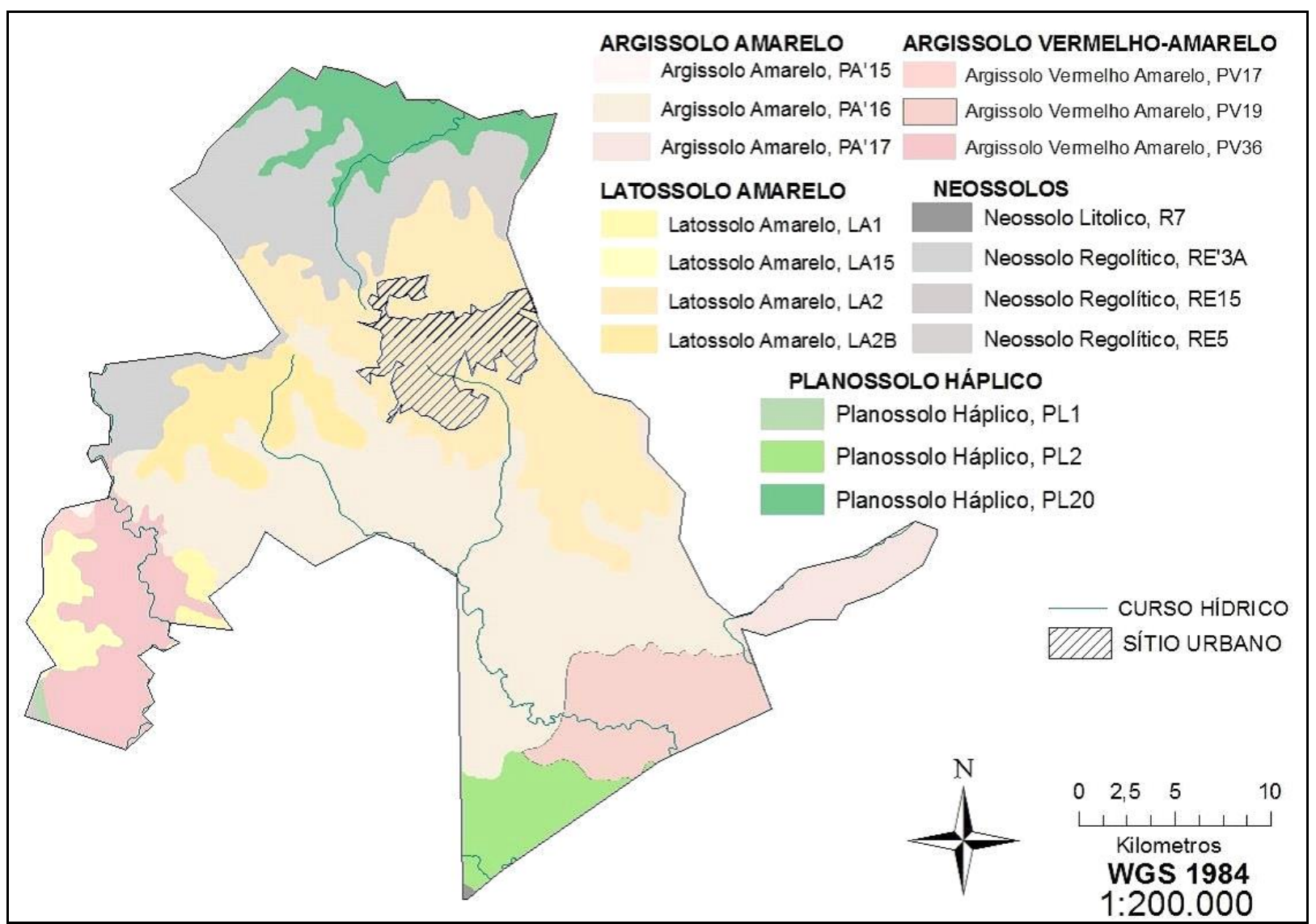

Figura 7: Mapa de solos do município de Garanhuns - PE. Fonte: Autor.

\section{CONSIDERAÇÕES FINAIS}

Os resultados deste trabalho aportam as primeiras informações quanto à dinâmica geomorfológica quaternária sobre os setores elevados do Planalto da Borborema em sua porção meridional. Os resultados apontaram para que os depósitos de encosta na área estão cronologicamente limitados entre o final do pleistoceno e o holoceno médio.

Constatou-se que a influência da dinâmica ambiental global, pontuada por flutuações climáticas regionais, atuou na elaboração do relevo da área de estudo, possibilitando, por meio da datação de sedimentos pelo método da Luminescência Opticamente Estimulada - LOE -, identificar os momentos em que a morfogênese atuou de forma mais ativa sobre as encostas, resultando na deposição de unidades coluviais.

A compreensão dos processos atuantes na área demanda a integração entre os aspectos paleoclimáticos e morfoestruturais da Cimeira Pernambuco-Alagoas, estando situada a salvaguarda das principais zonas de cisalhamentos regionais e, portanto, apresentando uma morfologia muito mais homogênea e aplainada, sobre a qual desenvolveram-se mantos de intemperismo mais espessos, sobretudo nas proximidades da escarpa oriental do Planalto.

Sobre o setor mais elevado desta unidade, designado como Patamar Erosivo de Garanhuns, os processos erosivos foram ativados e/ou intensificados pela precipitação mais elevada. A alternância entre fluxos superficiais concentrados e fluxos gravitacionais, a depender da intensidade dos regimes paleoclimáticos resultou ora no entrincheiramento da drenagem ao longo das encostas, ora na deposição de unidades coluviais. Por outro lado, as condições ambientais hodiernas associadas às práticas vigentes de uso e ocupação da terra resultam em ravinamentos generalizados sobre as unidades coluviais e cabeceiras de drenagem não canalizadas. 


\section{REFERÊNCIAS}

AITKEN, M. J. An introduction to optical dating. The dating of quaternary sediments by the use of photon-stimulated luminescence. Oxford, Oxford University Press: 267p, 1998.

AMORIM, R. F. Integração entre dinâmicas geomorfológicas multitemporais no planalto da Borborema, semiárido do NE do Brasil. Tese de Doutorado, Programa de Pós-graduação em Geografia, UFPE, $194 f$. 2015.

BARROS, L. C. Estudos sedimentológicos na plataforma continental interna adjacente às desembocaduras sul do canal de santa cruz e do rio Timbó, norte do estado de Pernambuco. Dissertação de Mestrado. Pós-Graduação em Geociência, Universidade Federal de Pernambuco - UFPE, Recife-PE, 2003, p. 42.

CHRISTOFOLETTI, A. Geomorfologia. São Paulo Ed. Edgard Blucher, p. 01-03, 1980.

CLARKE, M. L.; RENDELL, H. M.; WINTLE, A. G. Quality assurance in luminescence dating. Geomorphology, v.29, 1999, p.173-185.

COLTRINARI, L. Cartografia Geomorfológica Detalhada: A Representação Gráfica do Relevo entre 1950-1970. Revista Brasileira de Geomorfologia. V. 12, nº 3, 2011, p. 121-128.

CORRÊA, A. C. B. 2001. Dinâmica geomorfológica dos compartimentos elevados do Planalto da Borborema, Nordeste do Brasil. Universidade Estadual Paulista - UNESP, Rio Claro - SP. Tese de Doutorado. 386f.

CORRÊA, A. C. B., TAVAres, B. DE A. C., MONTEIRO, K. A., Cavalcanti, L. C. S., LIRA, D. R. Megageomorfologia e morfoestrutura do planalto da borborema. Revista do Instituto Geológico, v.31, 2010 , p.35 - 52.

DULlER, G. A. T., BØTTER-JENSEN, L., MURRAY, A. S. Combining infrared and green-laser stimulation sources in single-grain luminescence measurements of feldspar and quartz. Radiation Measurements, v.37, 2003, p.543-550.

Empresa Brasileira de Pesquisa Agropecuária - EMBRAPA. Sistema Brasileiro de Classificação de Solos. Ministério da Agricultura, Pecuária e Abastecimento. Governo Federal. $2^{\circ}$ Ed. Brasília, DF 2006, p.124-276.

FOLK, R. L. e WARD, W. Brazos River bar: a study in the significance of grain size parameters. Journal of Sedimentary Research, 27: 1957, 3-26.

GALVÃO, D. C. Reconstrução paleoambiental a partir dos colúvios do entorno da Lagoa do Puiu, Município de Ibimirim - Pernambuco. Dissertação (Mestrado em Geografia), Universidade Federal de Pernambuco, Recife. 2012, 130f.

GOUDIE, A. S. Encyclopedia of Geomorphology, London, Routledge, 2006, 1156 p.

GREGORY, K. J. e GOUDIE, A. S. (Eds.).The SAGE Handbook of Geomorphology. SAGE Pub USA, 2011, 610p.

GUEDES, C.C.F., SAWAKUCHI, A.O., GIANNINI, P.C.F., DEWITT, AGUIAR, V.A.P. Luminescence characteristics of quartz from Brazilian sediments and constraints for OSL dating. Anais da Academia Brasileira de Ciências, 85(4):1303-1316, 2013.

GUERRA, A.J.T. Encostas Urbanas. In:GUERRA, A. J. T.(Org.). Geomorfologia Urbana. Ed. Bertrand Brasil. Rio de Janeiro, 2011, p. 13 - 39.

LIMA, E. M. Evolução Paleoambiental do Município de Garanhuns-PE, a partir de Análises Sedimentológicas: Aplicação do Método de Luminescência Opticamente Estimulada - LOE. Universidade Federal de Pernambuco - UFPE, Dissertação de Mestrado, 2014, 105p.

LIMA, E. M., CORRÊA, A. C. B. Mapeamento geomorfológico como ferramenta de caracterização ambiental do Município de Garanhuns-PE. GEOSUL, Florianópolis-SC, 2016.

LIRA, D. R. Evolução geomorfológica e paleoambiental das Bacias do Riacho do Pontal e GI-8 no SubMédio São Francisco. Tese (doutorado) - Universidade Federal de Pernambuco, Recife. 2014. 234p.

MURRAY, A. S. e WINTLE, A. G. Luminescence dating of quartz using an improved single-aliquot regenerative-dose protocol. Radiation Measurement, v.32, 2000, p.57-73. 
PEIXOTO, M. N. O.; TATUMI, S. H.; MELLO, C. L.; BARRETO, A. M. F.; MOURA, J. R. S. Comparação entre idades obtidas por luminescência e idades radiocarbono de depósitos coluviais quaternários bananal e barra mansa (sp/rj), depressão do médio vale do rio paraíba do sul. $X$ Congresso da Associação Brasileira de Estudos do Quaternário. 2003, p. 01-04.

RENDELL, H. M.; WEBSTER, S. E.; SHEFFER, N. L. Underwater bleaching of signals from sediment grains: new experimental data. Quaternary Science Reviews, v.13, 1994, p.433-435.

SANTOS, M. L. Unidades Geomorfológicas e depósitos sedimentares associados no sistema fluvial do rio Paraná no seu curso superior. Revista Brasileira de Geomorfologia, Ano 6, No 1, 2005, p. 85-96.

SAWAKUCHI, A.O.; KALCHGRUBER, R.; GIANNINI, P.C.F.; NASCIMENTO JR, D.R.; GUEDES, C.C.F.; UMISEDO, N. The development of blowouts and foredunes in the Ilha Comprida barrier (Southeastern Brazil): the influence of Late Holocene climate changes on coastal sedimentation. Quaternary Science Reviews, 2008.

SCHMIDIT, C.; KREUTZER, S.; Optically stimuleted luminescence of amorphous/microcrystalline SiO2 (Silex): Basic investigation and potential in archeological dosimetry. Quaternary Geochronology, ed. Elsevier, 15:1-10, 2013.

SILVA, D. G. Evolução paleoambiental dos depósitos de tanques em Fazenda Nova, Município de Brejo da Madre de Deus, Pernambuco. Dissertação de Mestrado, Universidade federal de Pernambuco, 2007, $155 f$.

SUGUIO, K. Introdução à sedimentologia. São Paulo: Ed. da Universidade de São Paulo, 1973. 2010. Geologia do quaternário e mudanças ambientais. Ed. Oficina de Textos. São Paulo, SP,

TUCKER, M. Techniques in sedimentology. London, Blackwell. 1996.

WAYLAND, E.J. Peneplains and some other erosional plataforms. Ann. Rept. Bull. Protectorate of Uganda Geol Surv., note 1. 1933 p. 77-79.

MURRAY, A.S., WINTLE, A.G. Luminescence dating of quartz using an improved single-aliquot regenerative-dose protocol. Radiation Measurement, 2000. 32: 57-73.

WINTLE, A.G.; MURRAY, A.S.; A review of quartz optically stimulated luminescence characteristics and their relevance in single-aliquot regeneration dating protocols. Radiation Measurements, 2006. 41: 369-391. 